\title{
Plant hormone receptors: new perceptions
}

\author{
Angela K. Spartz and William M. Gray ${ }^{1}$ \\ Department of Plant Biology, University of Minnesota-Twin Cities, St. Paul, Minnesota 55108, USA
}

\begin{abstract}
Plant growth and development require the integration of a variety of environmental and endogenous signals that, together with the intrinsic genetic program, determine plant form. Central to this process are several growth regulators known as plant hormones or phytohormones. Despite decades of study, only recently have receptors for several of these hormones been identified, revealing novel mechanisms for perceiving chemical signals and providing plant biologists with a much clearer picture of hormonal control of growth and development.
\end{abstract}

The hormonal control of the growth and development of multicellular organisms has intrigued generations of biologists. In plants, virtually every aspect of the plant's life is regulated by a handful of small organic molecules collectively referred to as the phytohormones. Like animal hormones, the phytohormones act at very low concentrations and exert developmental control by regulating cell division, expansion, differentiation, and death. These effects can be highly complex, with a single cell responding to multiple hormones and a single hormone having differential effects on distinct tissues. Unlike the highly localized synthesis and transport via the circulatory or lymphatic systems characteristic of animal hormones, generally speaking, plant hormones are produced to various extents throughout the plant, and may elicit responses in the same cells or tissues where synthesis occurs. That said, phytohormones can be transported through the plant vasculature system or, at least in the case of auxin, through a complex cell-to-cell transport system that delivers auxin to its target cells in a highly regulated fashion (Vieten et al. 2007).

The origins of the concept of plant hormones can be traced to the late 19th century, when the German botanist Julian von Sachs proposed the existence of mobile endogenous compounds that act as specific "organ-forming substances." This coincided with a study by Charles and Francis Darwin on phototropism-the bending of plants toward light. The Darwins demonstrated that

[Keywords: Abscisic acid; auxin; gibberellin; jasmonate; phytohormones; receptors]

${ }^{1}$ Corresponding author.

E-MAIL grayx051@tc.umn.edu; FAX (612) 625-1738.

Article is online at http://www.genesdev.org/cgi/doi/10.1101/gad.1693208. when grass seedlings were exposed to a lateral light source, a transported signal originating from the plant apex promoted differential cell elongation in the lower parts of the seedling that resulted in it bending toward the light source. This signal was subsequently shown to be indole-3-acetic acid (IAA or auxin), the first known plant hormone. Once identified, the effects of each hormone were initially elucidated largely by observing the physiological responses elicited by exogenous applications. However, the identification of hormone biosynthesis and response mutants, particularly in the model plant Arabidopsis thaliana, has verified many of these roles, as well as established new functions for each hormone.

Central to comprehending hormonal control of plant growth and development is the understanding of how the hormones are perceived. In the past few years, tremendous progress has been achieved in elucidating the mechanisms of plant hormone perception. Here, we focus on recent progress in this field. We did not include discussion of hormone-binding proteins that have been isolated biochemically but for which there is little genetic support for receptor function. We also did not include discussion of the ethylene, cytokinin, or brassinosteroid receptors, as these receptors have a somewhat longer history, and instead refer readers to several excellent recent reviews (Kakimoto 2003; Benavente and Alonso 2006; Chow and McCourt 2006; Gendron and Wang 2007). While these three hormones are perceived by two-component regulators and receptor kinases, classes of proteins used by other organisms to communicate chemical signals, the receptors for the remaining phytohormones define novel perception mechanisms.

\section{Auxin: F-box proteins as receptors}

IAA (auxin) regulates an amazingly diverse array of processes in plant growth and development ranging from embryo patterning to growth responses to tropic stimuli (Woodward and Bartel 2005). Auxin response involves a large-scale reprogramming of gene expression affecting hundreds of auxin-regulated genes. The Arabidopsis TIR1 gene was identified more than a decade ago as a positive regulator of auxin response (Ruegger et al. 1998; Gray et al. 1999). TIR1 is one of several hundred F-box proteins encoded in the Arabidopsis genome. F-box pro- 
teins function as substrate recognition modules for multisubunit Skp1-Cullin1-F-box protein (SCF) ubiquitinligases (Petroski and Deshaies 2005). Since mutations in TIR1, as well as in the genes encoding the core SCF subunits, confer diminished auxin sensitivity, it seemed likely that the SCF ${ }^{\text {TIR1 }}$ complex might target negative regulators of auxin signaling for ubiquitin-mediated proteolysis. Such repressors became apparent through the combined approaches of forward and molecular genetics.

Aux/IAA genes were initially identified as rapid transcriptional targets of auxin action (Liscum and Reed 2002). The short-lived proteins encoded by these genes were subsequently found to interact with members of the AUXIN RESPONSE FACTOR (ARF) family of transcription factors that control the expression of many auxin-regulated genes, and cotransfection assays demonstrated that Aux/IAA proteins negatively regulate ARF transcriptional activity. Meanwhile, several laboratories identified dominant gain-of-function mutations in Aux/ $I A A$ genes in screens for mutants with reduced auxin response. All of the dominant lesions mapped to a highly conserved motif (domain II) that confers instability to Aux/IAA proteins. These findings raised the possibility that Aux/IAA proteins might be substrates of the $\mathrm{SCF}^{\mathrm{TIR} 1}$ complex. Indeed, Aux/IAA proteins interact with TIR1 and exhibit increased stability in mutants with impaired SCF ${ }^{\text {TIR1 }}$ function (Gray et al. 2001). In contrast, derivatives of Aux/IAA proteins containing mutations in domain II cannot interact with TIR1. Consequently, they exhibit increased stability, thus explaining the dominant, auxin-unresponsive nature of these mutants.

Of particular interest was the finding that auxin promotes the interaction between TIR 1 and its Aux/IAA substrates (Gray et al. 2001; Dharmasiri et al. 2003). The fact that auxin added to crude protein extracts enhanced the TIR1-Aux/IAA interaction raised the possibility that auxin signaling did not involve a complicated signaling cascade, but, rather, that auxin may bind directly to TIR1. At long last, the hunt for an auxin receptor concluded when the laboratories of Mark Estelle and Ottoline Leyser (Dharmasiri et al. 2005a; Kepinski and Leyser 2005) demonstrated that TIR1 binds auxin directly, which confers an increased affinity for Aux/IAA proteins. However, the fact that tir1 mutants exhibit only weak auxin response defects clearly indicated the presence of additional auxin receptors. Three additional Auxin F-Box (AFB) proteins sharing $\sim 60 \%-70 \%$ amino acid identity with TIR1 were subsequently found to bind Aux/IAA proteins in an auxin-dependent manner (Dharmasiri et al. 2005b). Consistent with the notion that the functions of the AFB and TIR1 proteins overlap, introduction of $a f b$ mutations into tir1 plants results in a progressive reduction in auxin response, culminating with tir1 afb1 afb2 afb3 quadruple mutants exhibiting a seedling lethal phenotype. Curiously however, a fraction of these quadruple mutants do survive and are able to complete development, suggesting that auxin signaling is not completely abolished in these mutants. Thus, auxin signaling appears startlingly simple and direct, with the hormone binding to the TIR1/AFB F-box proteins, thereby enabling their interaction with the Aux/ IAA repressors. The subsequent ubiquitin-mediated proteolysis of the Aux/IAAs derepresses the ARF transcription factors, leading to changes in auxin-regulated gene expression (Fig. 1A).

The TIR1 and AFB proteins are nuclear localized and exhibit typical F-box protein architecture, having an $\mathrm{N}$ terminal F-box domain that mediates interactions with the SKP1 SCF subunit, followed by a series of leucinerich repeats (LRRs) that comprise the substrate-binding domain. Crucial insight into how auxin regulates the interaction between TIR1 and its Aux/IAA substrates was recently obtained when the crystal structure of TIR1 bound to ASK1 (Arabidopsis SKP1) was solved with and without auxin and an Aux/IAA domain II peptide (Tan et al. 2007). The TIR1-ASK1 complex has the overall shape of a mushroom, with ASK1 and the F-box domain comprising the "stem" and the 18 LRRs folding into a closed, horseshoe-shaped solenoid to form the "cap." The auxinbinding site is formed by a hydrophobic pocket located on the upper surface of the LRR domain. IAA binds to the floor of this pocket, with the carboxyl group anchoring the hormone to the bottom of the pocket by forming a salt bridge and hydrogen bonds to polar residues. Meanwhile, the indole ring interacts with the sides of the pocket through hydrophobic and van der Waals attractions. The "ceiling" of the pocket is formed by the hydrophobic Aux/IAA degron peptide, which effectively traps auxin beneath it, presumably until the Aux/IAA protein is degraded. Auxin binding does not have an allosteric effect on TIR1, but, rather, fills a polar gap between TIR 1 and the Aux/IAA, thus extending the hydrophobic protein interaction surface, and acting as what Tan et al. (2007) describe as a "molecular glue." Thus, the optimal auxin-binding site requires the cooperative action of both TIR1 and the Aux/IAA protein. This is particularly interesting in light of previous studies, which have found considerable differences in the degradation rates of individual Aux/IAA family members (Dreher et al. 2006). It will be interesting to see if different TIR1/AFB-Aux/IAA combinations exhibit distinct IAA-binding affinities, and how this might contribute to the generation of developmentally specific auxin responses.

An unexpected surprise in the TIR1 crystal was the finding that a molecule of inositol hexakisphosphate $\left(\right.$ InsP $\left._{6}\right)$ copurified with the receptor. Although InsP $\mathrm{P}_{6}$ appears to be playing a structural role by coordinating several positively charged amino acids to support the floor of the auxin-binding pocket, a potential regulatory role has not been ruled out. In addition to the endogenous auxin IAA, TIR1 also binds to the synthetic auxins 2,4dichlorophenoxyacetic acid (2,4-D) and 1-naphthalene acetic acid (1-NAA), albeit with slightly lower affinities (Kepinski and Leyser 2005). While these compounds share the anchoring carboxyl group with IAA, the rings are significantly different. The structural studies of Tan et al. (2007) reveal that the slightly larger naphthalene ring of 1-NAA is oriented similarly to the indole ring of 


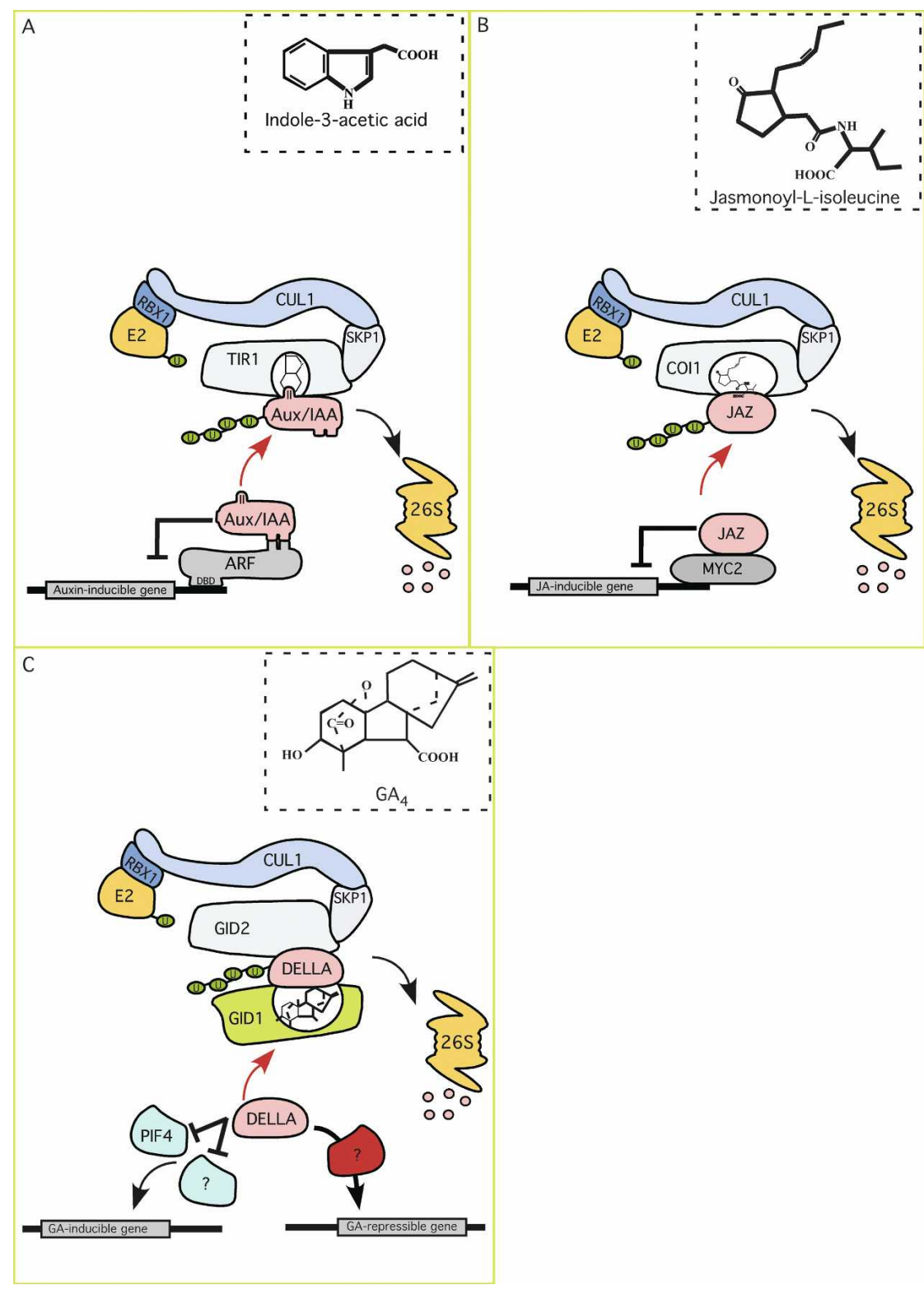

Figure 1. Ubiquitin-ligase based receptors. (A) Under subthreshold auxin concentrations, auxin-responsive genes are repressed by Aux/IAA proteins heterodimerizing with ARF transcription factors. Upon an auxin stimulus, the TIR1 F-box protein subunit of the $\mathrm{SCF}^{\mathrm{TIR} 1}$ ubiquitin-ligase binds auxin, enabling the recruitment of Aux/IAA proteins to the SCF complex for ubiquitination. Subsequent Aux/IAA degradation by the 26S proteasome derepresses the ARF transcription factors. $(B)$ JAZ proteins negatively regulate jasmonate response by repressing MYC2 transcriptional activity. Upon binding jasmonate, the $\mathrm{SCF}^{\mathrm{COI}}$ ubiquitin-ligase targets JAZ proteins for ubiquitin-mediated proteolysis, derepressing MYC2. (C) DELLA proteins repress GA response by negatively regulating PIF3, PIF4, and presumably other transcription factors that control the expression of GA-inducible genes. DELLA proteins also promote the expression of several GArepressible genes, several of which encode GA biosynthetic enzymes and components of the response pathway including the GID1 receptors. Upon binding GA, the GID1 receptor interacts with DELLA. The GID1GA-DELLA complex is then recognized by the $\mathrm{SCF}^{\mathrm{GID} 2}$ ubiquitin-ligase, which targets DELLA for ubiquitin-mediated degradation. The red arrows in $A-C$ indicate the effects of the respective hormone on the Aux/IAA, JAZ, and DELLA repressors.
IAA. However, IAA exhibits higher binding affinity presumably due to an additional hydrogen bond formed between the NH group on its ring and a nearby TIR1 carbonyl group. 2,4-D exhibits the weakest binding affinity because its smaller dichlorophenyl ring makes less surface contact with the partially promiscuous hydrophobic auxin-binding pocket.

Given the "molecular glue" hypothesis of how auxin facilitates interactions between TIR1 and its Aux/IAA substrates, it follows that a compound capable of occupying the TIR1 auxin-binding pocket but precluding Aux/IAA binding should exhibit anti-auxin activity. Hayashi et al. (2008) have recently taken a direct approach to identify such compounds. By introducing alkyl chains of increasing length to the $\alpha$-position of IAA and testing these compounds in a variety of auxin bioassays, the investigators found that IAA derivatives containing methyl-to-propyl additions retained auxin activity. However, the addition of longer side chains resulted in compounds that act as auxin antagonists. Crystallographic studies confirmed that the anti-auxin compounds occupy the auxin-binding pocket of TIR1 in the same way as IAA, with the alkyl chains extending upward into the Aux/IAA binding space. These structural studies were complemented with protein interaction assays demonstrating that anti-auxin binding to TIR1 blocks the TIR1-Aux/IAA interaction. Consistent with these findings, Arabidopsis plants treated with the antiauxin compounds exhibit increased Aux/IAA protein stability and several phenotypes indicative of reduced auxin response. Furthermore, anti-auxin treatment also diminished auxin response in rice and the moss Physcomitrella patens. While these distantly related plants are known to encode likely TIR1 and Aux/IAA ho- 
mologs, little is known about auxin response mechanisms in these species. The findings of Hayashi et al. (2008) strongly support the hypothesis that auxin perception and signal transduction are conserved throughout the plant kingdom. Thus, the anti-auxin probes will likely become a powerful new tool for studying auxinregulated growth and development in plants that lack the genetic tools provided by Arabidopsis, and potentially for manipulating auxin response in a developmental or organ-specific context for agricultural benefit.

The novelty in the mode of auxin perception raises several exciting possibilities. How widespread is this mechanism? Arabidopsis and rice both encode nearly 700 F-box proteins, nearly all of which remain to be characterized. Plants also possess much greater chemical complexity compared with animals, making it tempting to speculate that TIR1 may be the first of many F-box proteins that act as sensors for small signaling molecules. While most animal genomes encode many times fewer F-box proteins than plants, it is certainly conceivable that a similar ligand-binding substrate recruitment mechanism may regulate some animal ubiquitin-ligase activities as well. Regardless of whether or not the TIR1 receptor paradigm is unique to the plant kingdom, the structural studies described above have important implications beyond plants. Several human diseases have been associated with defects in ubiquitin-ligase-substrate interactions. Given the way that auxin promotes the interaction between TIR1 and its Aux/IAA substrates, it may be possible to design small therapeutic molecules that can either restore these interactions by acting as "molecular glue" or that block ubiquitin ligase function by preventing interaction with the substrate similar to the anti-auxin compounds described by Hayashi et al. (2008).

\section{Jasmonates: more of the same}

Collectively referred to as jasmonates, the oxylipin jasmonic acid and its metabolites regulate a variety of abiotic and biotic stress responses, as well as developmental processes including reproductive development and senescence (Devoto and Turner 2003). These responses are associated with changes in the expression of hundreds of downstream jasmonate-regulated genes via a signaling pathway that is remarkably similar to that of auxin. COI1, an F-box protein closely related to the TIR1/AFB family and that has been shown to interact with the core SCF subunits (Xu et al. 2002), has long been known to be required for jasmonate response (Xie et al. 1998). Recent findings suggest that in response to jasmonate, $\mathrm{SCF}^{\mathrm{COI} 1}$ targets members of the jasmonate ZIM domain (JAZ) protein family for ubiquitin-mediated degradation. The JAZ proteins were recently implicated as repressors of jasmonate response by three independent studies. Transcript profiling studies by Thines et al. (2007) and Yan et al. (2007) identified several $J A Z$ genes as being rapidly induced in response to jasmonate, while a forward genetic screen for jasmonate-insensitive mutants was used to isolate jai3-1, a dominant allele of $J A Z 3$ (Chini et al.
2007). The jai3-1 mutation affects a splice acceptor site that results in a truncated protein lacking a conserved C-terminal domain. Expression of other JAZ proteins containing C-terminal truncations also confers reduced jasmonate sensitivity. While this suggested that JAZ proteins might function as repressors, a clear interpretation was hampered by the finding that overexpression of fulllength JAZ proteins did not alter sensitivity to jasmonates. Likewise, possibly as a result of functional redundancy, individual null mutations of several $J A Z$ genes failed to confer any jasmonate phenotypes. However, the revelation that JAZ proteins are subject to degradation by the $26 \mathrm{~S}$ proteasome provides a likely explanation. Analysis of JAZ fusions with $\beta$-glucuronidase (GUS) or GFP reporter proteins revealed that multiple JAZ proteins are rapidly degraded in a COI1-dependent manner following jasmonate treatment. Importantly, JAZ proteins containing C-terminal deletions were resistant to degradation, suggesting that accumulation of these proteins is responsible for the jasmonate insensitivity conferred by these derivatives.

How do JAZ proteins repress jasmonate response? Like the Aux/IAA proteins of the auxin pathway, JAZ proteins lack a DNA-binding domain and appear to function by negatively regulating the activity of transcription factors. JAZ3 physically interacts with the MYC2 transcription factor that regulates the expression of many jasmonate-inducible genes, and the expression of many MYC2regulated genes is repressed in plants expressing the stabilized jai3-1 protein (Chini et al. 2007). Whether or not the other JAZ proteins also repress MYC2 or possibly other jasmonate-regulated transcription factors remains to be confirmed, but seems likely given the current available evidence.

The striking parallels between the jasmonate and auxin signaling pathways led to the suggestion that COIl functions as a jasmonate receptor, just as the closely related TIR1/AFB proteins do in the auxin pathway (Fig. 1B). This possibility was bolstered significantly by recent findings examining interactions between COI1 and the JAZ proteins. In both yeast two-hybrid and in vitro pull-down assays, the addition of biologically active jasmonates promoted the interaction between COI1 and the JAZ1 protein (Thines et al. 2007). Since COI1 and JAZ1 were the only plant proteins in the yeast twohybrid system, it seemed very likely that one or perhaps both proteins together act as the site of jasmonate perception. This possibility was further strengthened by the recent demonstration that coronatine also promotes the COI1-JAZ interaction and copurifies with this protein complex in pull-down assays from plant protein extracts (Katsir et al. 2008). Coronatine is a phytotoxin produced by certain plant pathogens that acts as a jasmonate molecular mimic. In these pull-down assays, the biologically active jasmonate JA-Ile competes with coronatine for binding to COI1-JAZ complexes, indicating that the two compounds are recognized by the same receptor. Thus, it appears that like TIR1, the COI1 F-box protein also functions as an intracellular receptor that stably binds its protein substrates only in the presence of its 
hormone ligand. Whether or not jasmonate acts to bridge the COI1-JAZ interaction in a manner similar to how auxin facilitates TIR1 binding to its substrates awaits the results of structural studies.

\section{Gibberellins (GA): variation of the ubiquitin-ligase-based receptor theme}

GA are tetracyclic, diterpenoid plant hormones that regulate many aspects of plant development including stem elongation, seed germination, and the induction of flowering (Fleet and Sun 2005). Like the auxin and jasmonate pathways, genetic screens in both rice and Arabidopsis for GA-insensitive mutants identified mutations in genes encoding F-box proteins (McGinnis et al. 2003; Sasaki et al. 2003). The rice GID2 and Arabidopsis SLY1 F-box proteins are closely related and have been shown to assemble into SCF ubiquitin-ligase complexes (McGinnis et al. 2003; Sasaki et al. 2003; Fu et al. 2004). In response to GA, SCF ${ }^{\mathrm{SLY} 1 / \mathrm{GID} 2}$ targets members of a family of proteins that repress GA responses, known as DELLA proteins, for ubiquitin-mediated proteolysis (Sasaki et al. 2003; Dill et al. 2004). DELLA proteins have long been thought to function as transcriptional regulators. Only recently, however, has direct evidence in support of this possibility been obtained. Two recent reports demonstrated that DELLAs interact with the DNA-binding domain of PIF3 and PIF4, two basic helix-loop-helix transcription factors that regulate cell expansion in response to light and GA. DELLA-binding sequesters these transcription factors from their target promoters, preventing transcriptional activation (de Lucas et al. 2008; Feng et al. 2008). Meanwhile, a third study used expression profiling to identify putative direct DELLA target genes (Zentella et al. 2007). All of the identified genes are GA-repressed and DELLA-induced, with several of them encoding GA biosynthesis enzymes, indicating a form of feedback regulation, while others encode potential negative regulators of GA signaling. Chromatin immunoprecipitation experiments detected DELLA association with the promoters of several of these genes, indicating that DELLAs can also regulate GA responses either by directly binding DNA or through other DNA-bound transcription factors. While the GA-dependent $\mathrm{SCF}^{\mathrm{SLY} 1 /}$ GID2-mediated ubiquitination of the DELLA repressors is very reminiscent of the auxin and jasmonate pathways discussed above, in this case, the F-box proteins are not hormone receptors.

Like gid2 mutants, rice plants containing mutations in gid1 are unresponsive to GA and are unable to degrade the DELLA domain repressor SLR1 (Ueguchi-Tanaka et al. 2005). Double mutant studies positioned GID1 upstream of $S L R 1$, and the recombinant protein was found to bind bioactive GAs in vitro with both high specificities and affinities, consistent with GID1 functioning as the GA receptor. Predominantly localized to the nucleus, the GID1 protein exhibits sequence similarity to animal hormone-sensitive lipases (HSL). While mutations in the HSL-related domain abolish GID1 function, it does not appear to function as a lipase, as the protein shares only two of three essential residues required for HSL activity and cannot hydrolyze an artificial HSL substrate in vitro.

A connection to the DELLA repressors of GA signaling was established by the demonstration that GA binding promotes a protein-protein interaction between GID1 and the SLR1 DELLA domain protein in both yeast twohybrid assays and in planta (Ueguchi-Tanaka et al. 2005, 2007). Interestingly, the addition of SLR1 to in vitro GAbinding assays increases GID1's binding activity by reducing the dissociation rate between the hormone and GID1. This raises the possibility that SLR1 binding to GID1 may "trap" GA in the GID1-binding site similarly to the way Aux/IAA proteins stabilize the interaction between TIR1 and auxin.

While the rice studies suggest how the GA signal may be transduced from the GID1 receptor to SLR1, how does $\mathrm{SCF}^{\mathrm{SLY} 1 / \mathrm{GID} 2}$ regulate DELLA protein stability? A recent study on the orthologous components from Arabidopsis suggests a potential mechanism. Unlike rice, which contains single copies of GID1 and SLR1, the Arabidopsis genome encodes three GID1 and five DELLA proteins. Like rice GID1, the three Arabidopsis receptors all exhibit high affinity for biologically active GAs and interact with DELLA proteins in a hormone-dependent manner (Nakajima et al. 2006). Genetic studies indicate that these receptors exhibit overlapping function, as single mutants develop normally, whereas double mutant combinations display a partial reduction in GA sensitivity, and the triple mutant plants are severely dwarfed and unresponsive to GA (Griffiths et al. 2006; Willige et al. 2007). Likewise, the five DELLA proteins appear partially redundant with one another, although several studies indicate that the individual DELLA members also display some specificity in terms of which GA responses they primarily control. Consistent with the hypothesis that $\mathrm{SCF}^{\mathrm{SLY} 1 / \mathrm{GID} 2}$ targets DELLA proteins for ubiquitin-mediated proteolysis, Griffiths et al. (2006) recently demonstrated that the SLY1 F-box protein can interact, albeit weakly, with the RGA DELLA domain protein in a yeast two-hybrid system. However, this interaction is dramatically enhanced in a GA-dependent manner when the GIDla receptor is added in a threehybrid assay. This suggests a model where upon binding GA, the receptor interacts with the DELLA protein, and the GID1-GA-DELLA complex is then recognized by $\mathrm{SCF}^{\mathrm{SLY} 1 / \mathrm{GID} 2}$, with the ensuing ubiquitination and degradation of the DELLA repressor leading to derepression of GA responses (Fig. 1C). The existence of a GID1-GADELLA complex in planta and how such a complex might interact with $\mathrm{SCF}^{\mathrm{SLY} 1 / \mathrm{GID} 2}$ remains to be determined.

\section{Abscisic acid: multiple receptors and pathways}

Abscisic acid (ABA) regulates a diverse array of processes including seed maturation and dormancy, root growth, leaf senescence, and the transition between vegetative and reproductive growth. It is also a major stress hormone that regulates the adaptation of plants to environ- 
mental stresses including cold, drought, and salt by modulating the expression of stress-responsive genes and controlling the aperture of stomates to regulate water content. Genetic studies have implicated a dizzying array of genes in ABA signaling including kinases, phosphatases, G-proteins, ubiquitin pathway components, RNA processing enzymes, and transcription factors. Several second messengers such as $\mathrm{Ca}^{2+}$, reactive oxygen species, nitric oxide, and phospholipids also appear to play roles in some ABA responses. Several recent reviews on ABA signaling discuss these signaling components in more detail (Himmelbach et al. 2003; Israelsson et al. 2006; Hirayama and Shinozaki 2007). Given this diversity of potential signaling components, perhaps it should not be surprising that in the past few years, three distinct ABA receptors have been identified.

The first reported ABA receptor was the FLOWERING TIME CONTROL PROTEIN A (FCA), a factor previously implicated in the vegetative-to-reproductive transition (Razem et al. 2006). Unlike the receptors discussed thus far, this receptor was isolated biochemically by screening a barley cDNA library for proteins exhibiting ABA-binding activity in vitro. Sequence analysis of the barley protein revealed that it was highly related to the Arabidopsis FCA protein, which was subsequently shown to also bind ABA. FCA specifically and reversibly binds physiologically active $(+)$ ABA, and binding affinity is sufficiently high $\left(K_{\mathrm{d}}=19 \mathrm{nM}\right)$ for the protein to sense physiological concentrations of the hormone.

Originally isolated as a late-flowering mutant, FCA encodes a nuclear localized RNA-binding protein that physically interacts with the RNA 3 '-end processing factor FY (Simpson et al. 2004). Together, FCA and FY control the processing of FCA, and presumably other, transcripts by promoting premature cleavage and polyadenylation of the pre-mRNA, resulting in production of a nonfunctional FCA protein. By a mechanism that remains to be elucidated, FCA and FY prevent the accumulation of FLC transcripts, which encode a MADS-box transcription factor that inhibits flowering. One simple possibility is that the FCA-FY complex targets the FLC pre-mRNA similarly to the FCA autoregulation, but this remains to be seen. Importantly, Razem et al. (2006) connect $\mathrm{ABA}$ to this control of flowering. The binding of ABA to FCA severely inhibited the ability of FCA to interact with FY in in vitro pull-down assays. Furthermore, ABA application to wild-type plants caused a delay in flowering and changes in the FCA and FLC transcript profiles similar to $f c a$ and $f y$ mutants, providing strong support for the hypothesis that FCA functions as an ABA receptor to regulate flowering time.

As an RNA-binding protein, FCA defines an entirely new class of hormone receptors. On a very basic level, however, the effects of ABA binding to the FCA receptor are similar to the auxin, jasmonate, and GA receptors described above, with hormone binding regulating a direct interaction between the receptor and another component of a seemingly very short signaling pathway. Equally clear from this study, however, is that FCA is not the only ABA receptor. $f c a$ mutants do not exhibit defects in seed germination or stomatal opening, two well-characterized ABA responses. Furthermore, mutations in $A B I 1$ and $A B I 2$, two protein phosphatases that have been implicated previously in ABA signaling, do not confer defects in the FCA-mediated control of flowering time or FLC abundance, suggesting the possibility of multiple ABA signaling pathways.

Another equally surprising ABA-binding protein, designated $A B A R$, was first purified from epidermal cells of broad bean leaves (Zhang et al. 2002). Recently, sequence analysis identified the ABAR gene. It encodes the putative $\mathrm{H}$ subunit (CHLH) of the chloroplast enzyme, magnesium-protoporphyrin-IX chelatase (Shen et al. 2006). In addition to catalyzing the first step unique to chlorophyll biosynthesis, CHLH has been implicated previously in retrograde signaling to control the expression of nuclear genes encoding plastid proteins (Mochizuki et al. 2001; Strand et al. 2003). However, initial studies suggest that the ABA-binding activity of CHLH may be independent of the roles it plays in chlorophyll synthesis and retrograde signaling. Genetic support for CHLH functioning as an ABA receptor was provided by characterizing Arabidopsis plants with increased or decreased CHLH levels (Shen et al. 2006). Plants in which CHLH was knocked down with an RNAi construct exhibited reduced sensitivity to $\mathrm{ABA}$ in germination, stomatal closure, and gene expression assays, as does a CHLH mutant containing a missense mutation that moderately diminishes ABA-binding activity. In contrast, plants overexpressing CHLH displayed ABA hypersensitivity in these assays. These data strongly support the conclusion that $\mathrm{CHLH}$ is a positive regulator of ABA signaling. However, the molecular mechanisms by which CHLH might transduce ABA signals remain to be elucidated.

While FCA and CHLH are confined to the nucleus and plastid, respectively, earlier studies have provided substantial evidence for an extracellular ABA receptor (Anderson et al. 1994; Gilroy and Jones 1994; Jeannette et al. 1999). The Arabidopsis RECEPTORLIKE KINASE1 (RPK1) protein is localized to the plasma membrane, and mutants exhibit reduced ABA sensitivity, raising the possibility that it may be an extracellular receptor (Osakabe et al. 2005). However, ABA binding has yet to be demonstrated. Arabidopsis G-protein mutants have ABA response defects, suggesting that a G-protein-coupled receptor (GPCR) might function as a plasma membrane-localized site of perception. GCR1 and RGS1 encode two candidate GPCRs, and genetic studies have implicated both in ABA response (Pandey and Assmann 2004; Chen et al. 2006; Pandey et al. 2006). Once again, however, ABA binding to these proteins has not been shown. Liu et al. (2007) recently examined a third putative GPCR, designated GCR2, and found that this protein binds ABA with the stereospecificity and affinity expected of a receptor.

Canonical G protein signaling involves a seven-transmembrane GPCR that interacts with a heterotrimeric G-protein consisting of $\alpha, \beta$, and $\gamma$ subunits. Upon ligand binding to the receptor, the G $\alpha$ subunit releases GDP and binds GTP, and dissociates from the G $\beta \gamma$ dimer. 
Subsequently, both G $\alpha$ and G $\beta \gamma$ are then free to interact with downstream effectors to pass on the signal. When GTP is hydrolyzed by the intrinsic GTPase activity of $\mathrm{G} \alpha$, the trimer is reconstituted and reassociates with the receptor. In plants, however, much less is known about G-protein signaling (Temple and Jones 2007). Arabidopsis encodes only one $\mathrm{G} \alpha$ subunit (GPA1), one G $\beta$ subunit, (AGB1), and two Gy subunits (AGG1 and $A G G 2$ ). Furthermore, unlike the hundreds of GPCRs found in animals, Arabidopsis encodes many fewer potential GPCRs, most of which are highly divergent and none of which has been assigned a ligand. Plants also appear to lack adenyl cyclase and phospholipase $C \beta$, two of the most common effectors of G-protein signaling pathways in animals. Thus it is unclear at this point whether plants use canonical or a variant form of GPCR-mediated G-protein signaling. The recent finding that Arabidopsis plants lacking $\mathrm{G} \gamma$ function respond normally to ABA, whereas both $\mathrm{G} \alpha$ and $\mathrm{G} \beta$ mutants exhibit several ABA response defects, strongly suggests that, at least in the case of ABA signaling, a noncanonical form of G-protein signaling is used (Trusov et al. 2008).

Liu et al. (2007) reported that mutations in the putative GPCR protein encoded by GCR2 conferred moderately reduced sensitivity to ABA in several standard assays including seed germination, stomatal movements, ABA-regulated gene expression, and $\mathrm{K}^{+}$channel activity in guard cells. Furthermore, the GCR2 protein localized to plasma membranes and interacts with the $\mathrm{G} \alpha$ subunit of heterotrimeric G-proteins, and, when expressed in yeast, the $\mathrm{G} \alpha$ interaction was modestly reduced upon ABA treatment. These findings, together with the ABAbinding activity of GCR2, are consistent with the notion that GCR2 functions as an ABA receptor upstream of a G-protein signaling pathway. However, recent work has raised some serious questions with this model. First, further bioinformatics analyses have suggested that GCR2 does not contain the characteristic seven-transmembrane spans typical of GPCRs, but rather is more closely related to lanthionine synthetase, a peripheral membrane protein (Gao et al. 2007; Johnston et al. 2007). More troublesome, however, is that, using the same gcr2 alleles originally described by Liu et al. (2007), Gao et al. (2007) were unable to observe significant differences in ABA sensitivity from wild-type in germination and ABAregulated gene expression assays. Stomatal movements and guard cell channel activity were not assessed in this most recent study. Lastly, with the exception of $\mathrm{G} \alpha$ acting as a positive regulator of stomatal opening, genetic studies of $\mathrm{G} \alpha$ and $\mathrm{G} \beta$ mutants indicate that $\mathrm{G}$-proteins negatively control ABA responses. This conflicts with the positive role GCR2 has been proposed to play in mediating ABA signals (Liu et al. 2007). Clearly, the finding that ABA binds the GPCR-like GCR2 protein is an exciting development. However, the discrepancies in the ABA sensitivity of gcr2 mutants and the potential signaling mechanisms between GCR2 and G-proteins remain to be resolved.

The identification of three unrelated receptors in three different cellular locales paints a very complicated picture of ABA signaling (Fig. 2). Instead of a single receptor acting upstream of a branched signaling pathway, these findings suggest that ABA responses are mediated by partially if not entirely distinct receptor classes and signaling components. Establishing molecular connections between the receptors and the wealth of ABA signaling

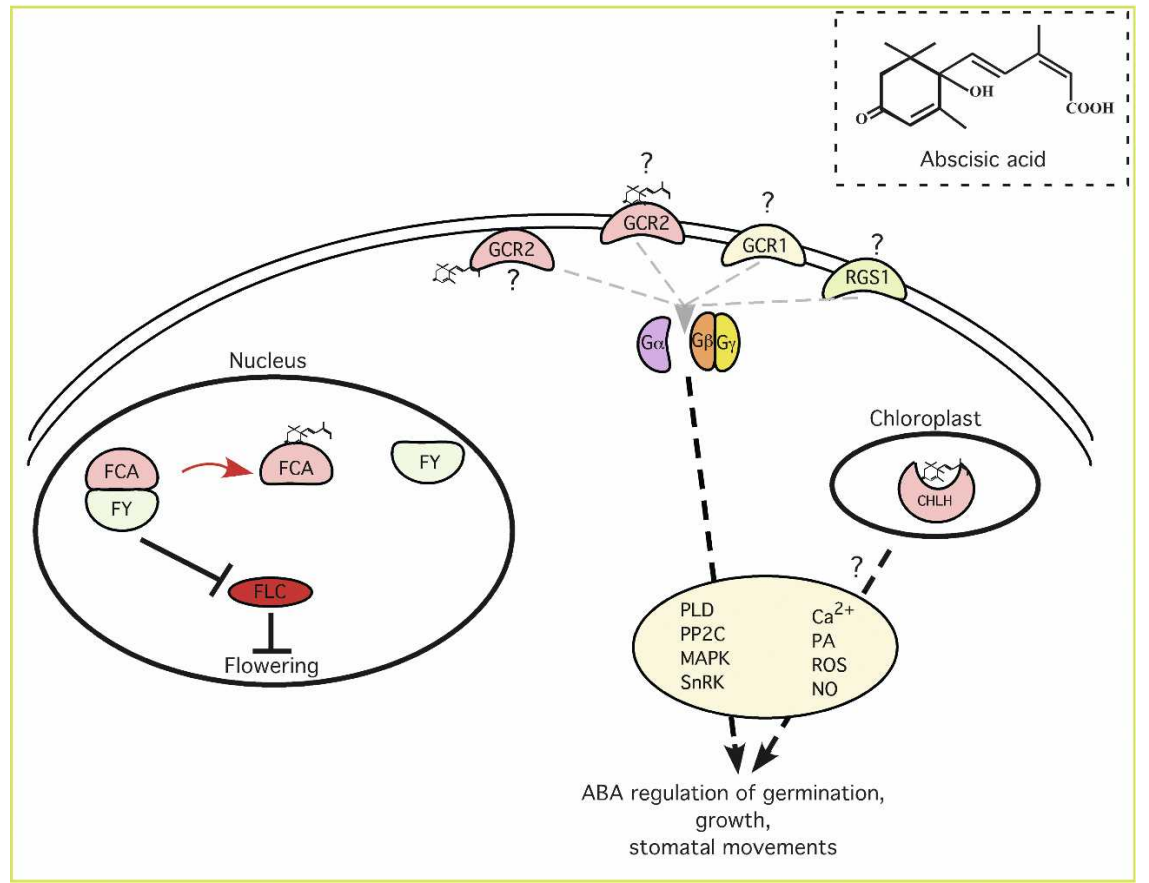
SNF1-related kinase; (PA) phosphatidic acid; (ROS) reactive oxygen species; (NO) nitric oxide.

Figure 2. ABA receptors act at different cellular locations. The RNA-binding protein FCA acts in the nucleus together with the mRNA 3 '-end processing factor FY to control the abundance of FLC, an inhibitor of flowering. Upon binding ABA, FCA and FY dissociate, leading to the accumulation of FLC and inhibition of flowering. In the chloroplast, the $\mathrm{Mg}$-chelatase $\mathrm{H}$ subunit (CHLH) binds to ABA and acts as a positive regulator of $\mathrm{ABA}$ signaling by an unknown mechanism. This function appears to be separable from $\mathrm{CHLH}^{\prime}$ s role in plastid-tonucleus signaling and chlorophyll synthesis. GPCRs have been proposed to function as ABA receptors on the plasma membrane. Genetic studies have implicated GCR1 and RGS1 as potential ABA receptors, but ABA binding activity has not been demonstrated. The GPCR-related protein GCR2 does bind ABA. However, it is currently unclear whether or not GCR2 is a canonical serpentine GPCR or a peripheral membrane protein. Genetic studies have implicated G-proteins and several potential downstream effectors and secondary ; (MAPK) mitogen-activated kinase; (SnRK) oxide.
oxide 
components identified through genetic studies, and identifying potentially common nodes connecting the individual pathways will hopefully begin to clarify this picture in the near future.

\section{Concluding remarks}

With a flurry of activity in the past few years, receptors for all of the classical plant hormones have now been identified. Unlike the previously identified receptors for ethylene, cytokinin, and brassinosteroids, these most recent additions to the plant hormone receptor family define highly novel perception mechanisms that have not yet been reported in animal or microbial systems. That said, not all animal hormones are perceived by classic types of receptors. For example, hepcidin, a peptide hormone regulating iron homeostasis is perceived by the cellular iron effluxor ferroportin (De Domenico et al. 2007). Hepcidin binding triggers the internalization, ubiquitination, and degradation of ferroportin, resulting in a reduction in serum iron levels. In terms of its apparent simplicity, such a regulatory system is somewhat reminiscent of the signaling mechanisms used by several of the phytohormone receptors discussed above, which appear to involve a very limited number of signaling components. A significant difference between hepcidin and the phytohormones, however, lies in the complexity of the response. Unlike hepcidin, the plant hormones regulate numerous and diverse responses, often in an organ- or cell-specific manner. While a short signaling pathway would enable a very rapid response, this would seem to limit the plant's ability to tightly control the response through feedback loops between signaling steps or to integrate signaling information from other pathways, such as is seen in the kinase cascades of many other signaling pathways. Rather, our current signaling models for several of the plant hormones resemble "OnOff" switches rather than tightly buffered signaling pathways. Such a mechanism might work fine for a receptor such as FCA, which appears to specifically transduce signals for only a small subset of ABA responses. However, it is much less clear how, from the myriad of possible responses to auxin, for example, the TIR1/AFB receptors promote a specific response within a given developmental context. The simplicity of current models is likely a reflection that our understanding of hormone signaling mechanisms is far from complete. Genetic and physiology studies have identified interactions between individual hormones as well as with other signaling pathways, although in most cases the molecular connections have yet to be elucidated. Furthermore, for many of the hormones, feedback loops between the signaling, metabolism, and transport pathways have been established. Determining how these feedback controls and the integration of signals from multiple pathways generate specific hormone responses in a cell/tissue-dependent manner remains a major challenge. Whether this specificity is achieved primarily through cell/tissue-specific differences in the expression of downstream transcription factors or additional, undiscovered layers of regulatory com- plexity on the receptors or signaling components themselves remains to be determined. However, having the receptors in hand provides a conceptual framework and novel tools that will undoubtedly greatly facilitate the dissection of the complex roles of hormones in plant growth and development.

\section{Acknowledgments}

We thank Dr. Neil Olszewski for his comments on the manuscript. We apologize to the authors whose work we were not able to cite due to space constraints. We acknowledge support from the National Institutes of Health and the National Science Foundation.

\section{References}

Anderson, B.E., Ward, J.M., and Schroeder, J.I. 1994. Evidence for an extracellular reception site for abscisic acid in commelina guard cells. Plant Physiol. 104: 1177-1183.

Benavente, L.M. and Alonso, J.M. 2006. Molecular mechanisms of ethylene signaling in Arabidopsis. Mol. Biosyst. 2: 165173.

Chen, Y., Ji, F., Xie, H., Liang, J., and Zhang, J. 2006. The regulator of G-protein signaling proteins involved in sugar and abscisic acid signaling in Arabidopsis seed germination. Plant Physiol. 140: 302-310.

Chini, A., Fonseca, S., Fernandez, G., Adie, B., Chico, J.M., Lorenzo, O., Garcia-Casado, G., Lopez-Vidriero, I., Lozano, F.M., Ponce, M.R., et al. 2007. The JAZ family of repressors is the missing link in jasmonate signalling. Nature 448: 666671.

Chow, B. and McCourt, P. 2006. Plant hormone receptors: Perception is everything. Genes \& Dev. 20: 1998-2008.

De Domenico, I., Ward, D.M., Langelier, C., Vaughn, M.B., Nemeth, E., Sundquist, W.I., Ganz, T., Musci, G., and Kaplan, J. 2007. The molecular mechanism of hepcidin-mediated ferroportin down-regulation. Mol. Biol. Cell 18: 2569-2578.

de Lucas, M., Daviere, J.M., Rodriguez-Falcon, M., Pontin, M., Iglesias-Pedraz, J.M., Lorrain, S., Fankhauser, C., Blazquez, M.A., Titarenko, E., and Prat, S. 2008. A molecular framework for light and gibberellin control of cell elongation. $\mathrm{Na}$ ture 451: 480-484.

Devoto, A. and Turner, J.G. 2003. Regulation of jasmonate-mediated plant responses in Arabidopsis. Ann. Bot. (Lond.) 92: 329-337.

Dharmasiri, N., Dharmasiri, S., Jones, A.M., and Estelle, M. 2003. Auxin action in a cell-free system. Curr. Biol. 13: $1418-1422$.

Dharmasiri, N., Dharmasiri, S., and Estelle, M. 2005a. The Fbox protein TIR1 is an auxin receptor. Nature 435: 441-445.

Dharmasiri, N., Dharmasiri, S., Weijers, D., Lechner, E., Yamada, M., Hobbie, L., Ehrismann, J.S., Jurgens, G., and Estelle, M. 2005b. Plant development is regulated by a family of auxin receptor F box proteins. Dev. Cell 9: 109-119.

Dill, A., Thomas, S.G., Hu, J., Steber, C.M., and Sun, T.P. 2004. The Arabidopsis F-box protein SLEEPY1 targets gibberellin signaling repressors for gibberellin-induced degradation. Plant Cell 16: 1392-1405.

Dreher, K.A., Brown, J., Saw, R.E., and Callis, J. 2006. The Arabidopsis Aux/IAA protein family has diversified in degradation and auxin responsiveness. Plant Cell 18: 699-714.

Feng, S., Martinez, C., Gusmaroli, G., Wang, Y., Zhou, J., Wang, F., Chen, L., Yu, L., Iglesias-Pedraz, J.M., Kircher, S., et al. 
2008. Coordinated regulation of Arabidopsis thaliana development by light and gibberellins. Nature 451: 475-479.

Fleet, C.M. and Sun, T.P. 2005. A DELLAcate balance: The role of gibberellin in plant morphogenesis. Curr. Opin. Plant Biol. 8: 77-85.

Fu, X., Richards, D.E., Fleck, B., Xie, D., Burton, N., and Harberd, N.P. 2004. The Arabidopsis mutant sleepylgar2-1 protein promotes plant growth by increasing the affinity of the SCFSLY1 E3 ubiquitin ligase for DELLA protein substrates. Plant Cell 16: 1406-1418.

Gao, Y., Zeng, Q., Guo, J., Cheng, J., Ellis, B.E., and Chen, J.G. 2007. Genetic characterization reveals no role for the reported ABA receptor, GCR2, in ABA control of seed germination and early seedling development in Arabidopsis. Plant J. 52: 1001-1013.

Gendron, J.M. and Wang, Z.Y. 2007. Multiple mechanisms modulate brassinosteroid signaling. Curr. Opin. Plant Biol. 10: $436-441$.

Gilroy, S. and Jones, R.L. 1994. Perception of gibberellin and abscisic acid at the external face of the plasma membrane of barley (Hordeum vulgare L.) aleurone protoplasts. Plant Physiol. 104: 1185-1192.

Gray, W.M., del Pozo, J.C., Walker, L., Hobbie, L., Risseeuw, E., Banks, T., Crosby, W.L., Yang, M., Ma, H., and Estelle, M. 1999. Identification of an SCF ubiquitin-ligase complex required for auxin response in Arabidopsis thaliana. Genes \& Dev. 13: 1678-1691.

Gray, W.M., Kepinski, S., Rouse, D., Leyser, O., and Estelle, M. 2001. Auxin regulates SCFTIR1-dependent degradation of AUX/IAA proteins. Nature 414: 271-276.

Griffiths, J., Murase, K., Rieu, I., Zentella, R., Zhang, Z.L., Powers, S.J., Gong, F., Phillips, A.L., Hedden, P., Sun, T.P., et al. 2006. Genetic characterization and functional analysis of the GID1 gibberellin receptors in Arabidopsis. Plant Cell 18: 3399-3414.

Hayashi, K., Tan, X., Zheng, N., Hatate, T., Kimura, Y., Kepinski, S., and Nozaki, H. 2008. Small-molecule agonists and antagonists of F-box protein-substrate interactions in auxin perception and signaling. Proc. Natl. Acad. Sci. 105: 56325637.

Himmelbach, A., Yang, Y., and Grill, E. 2003. Relay and control of abscisic acid signaling. Curr. Opin. Plant Biol. 6: 470-479.

Hirayama, T. and Shinozaki, K. 2007. Perception and transduction of abscisic acid signals: Keys to the function of the versatile plant hormone ABA. Trends Plant Sci. 12: 343-351.

Israelsson, M., Siegel, R.S., Young, J., Hashimoto, M., Iba, K., and Schroeder, J.I. 2006. Guard cell ABA and CO2 signaling network updates and $\mathrm{Ca}^{2+}$ sensor priming hypothesis. Curr. Opin. Plant Biol. 9: 654-663.

Jeannette, E., Rona, J.P., Bardat, F., Cornel, D., Sotta, B., and Miginiac, E. 1999. Induction of RAB18 gene expression and activation of $\mathrm{K}^{+}$outward rectifying channels depend on an extracellular perception of ABA in Arabidopsis thaliana suspension cells. Plant $J$. 18: 13-22.

Johnston, C.A., Temple, B.R., Chen, J.G., Gao, Y., Moriyama, E.N., Jones, A.M., Siderovski, D.P., and Willard, F.S. 2007. Comment on 'A G protein coupled receptor is a plasma membrane receptor for the plant hormone abscisic acid.' Science 318: 914.

Kakimoto, T. 2003. Perception and signal transduction of cytokinins. Annu. Rev. Plant Biol. 54: 605-627.

Katsir, L., Schilmiller, A.L., Staswick, P.E., He, S.Y., and Howe, G.A. 2008. COI1 is a critical component of a receptor for jasmonate and the bacterial virulence factor coronatine. Proc. Nat1. Acad. Sci. 105: 7100-7105.

Kepinski, S. and Leyser, O. 2005. The Arabidopsis F-box protein
TIR1 is an auxin receptor. Nature 435: 446-451.

Liscum, E. and Reed, J.W. 2002. Genetics of Aux/IAA and ARF action in plant growth and development. Plant Mol. Biol. 49: 387-400.

Liu, X., Yue, Y., Li, B., Nie, Y., Li, W., Wu, W.H., and Ma, L. 2007. A G protein-coupled receptor is a plasma membrane receptor for the plant hormone abscisic acid. Science 315: $1712-1716$

McGinnis, K.M., Thomas, S.G., Soule, J.D., Strader, L.C., Zale, J.M., Sun, T.P., and Steber, C.M. 2003. The Arabidopsis SLEEPY1 gene encodes a putative F-box subunit of an SCF E3 ubiquitin ligase. Plant Cell 15: 1120-1130.

Mochizuki, N., Brusslan, J.A., Larkin, R., Nagatani, A., and Chory, J. 2001. Arabidopsis genomes uncoupled 5 (GUN5) mutant reveals the involvement of $\mathrm{Mg}$-chelatase $\mathrm{H}$ subunit in plastid-to-nucleus signal transduction. Proc. Natl. Acad. Sci. 98: 2053-2058.

Nakajima, M., Shimada, A., Takashi, Y., Kim, Y.C., Park, S.H., Ueguchi-Tanaka, M., Suzuki, H., Katoh, E., Iuchi, S., Kobayashi, M., et al. 2006. Identification and characterization of Arabidopsis gibberellin receptors. Plant J. 46: 880-889.

Osakabe, Y., Maruyama, K., Seki, M., Satou, M., Shinozaki, K., and Yamaguchi-Shinozaki, K. 2005. Leucine-rich repeat receptor-like kinasel is a key membrane-bound regulator of abscisic acid early signaling in Arabidopsis. Plant Cell 17: 1105-1119.

Pandey, S. and Assmann, S.M. 2004. The Arabidopsis putative $\mathrm{G}$ protein-coupled receptor GCR1 interacts with the $\mathrm{G}$ protein $\alpha$ subunit GPA1 and regulates abscisic acid signaling. Plant Cell 16: 1616-1632.

Pandey, S., Chen, J.G., Jones, A.M., and Assmann, S.M. 2006. G-protein complex mutants are hypersensitive to abscisic acid regulation of germination and postgermination development. Plant Physiol. 141: 243-256.

Petroski, M.D. and Deshaies, R.J. 2005. Function and regulation of cullin-RING ubiquitin ligases. Nat. Rev. Mol. Cell Biol. 6: 9-20.

Razem, F.A., El-Kereamy, A., Abrams, S.R., and Hill, R.D. 2006. The RNA-binding protein FCA is an abscisic acid receptor. Nature 439: 290-294.

Ruegger, M., Dewey, E., Gray, W.M., Hobbie, L., Turner, J., and Estelle, M. 1998. The TIR1 protein of Arabidopsis functions in auxin response and is related to human SKP2 and yeast grrlp. Genes \& Dev. 12: 198-207.

Sasaki, A., Itoh, H., Gomi, K., Ueguchi-Tanaka, M., Ishiyama, K., Kobayashi, M., Jeong, D.H., An, G., Kitano, H., Ashikari, M., et al. 2003. Accumulation of phosphorylated repressor for gibberellin signaling in an F-box mutant. Science 299: 1896-1898.

Shen, Y.Y., Wang, X.F., Wu, F.Q., Du, S.Y., Cao, Z., Shang, Y., Wang, X.L., Peng, C.C., Yu, X.C., Zhu, S.Y., et al. 2006. The $\mathrm{Mg}$-chelatase $\mathrm{H}$ subunit is an abscisic acid receptor. Nature 443: 823-826.

Simpson, G.G., Quesada, V., Henderson, I.R., Dijkwel, P.P., Macknight, R., and Dean, C. 2004. RNA processing and Arabidopsis flowering time control. Biochem. Soc. Trans. 32: 565-566.

Strand, A., Asami, T., Alonso, J., Ecker, J.R., and Chory, J. 2003. Chloroplast to nucleus communication triggered by accumulation of Mg-protoporphyrinIX. Nature 421: 79-83.

Tan, X., Calderon-Villalobos, L.I., Sharon, M., Zheng, C., Robinson, C.V., Estelle, M., and Zheng, N. 2007. Mechanism of auxin perception by the TIR1 ubiquitin ligase. Nature 446: 640-645.

Temple, B.R. and Jones, A.M. 2007. The plant heterotrimeric G-protein complex. Annu. Rev. Plant Biol. 58: 249-266. 
Thines, B., Katsir, L., Melotto, M., Niu, Y., Mandaokar, A., Liu, G., Nomura, K., He, S.Y., Howe, G.A., and Browse, J. 2007. JAZ repressor proteins are targets of the SCF(COI1) complex during jasmonate signalling. Nature 448: 661-665.

Trusov, Y., Zhang, W., Assmann, S.M., and Botella, J.R. 2008. $G \gamma 1+G \gamma 2$ ! $=G \beta$ : Heterotrimeric G protein $G \gamma$-deficient mutants do not recapitulate all phenotypes of $\mathrm{G} \beta$-deficient mutants. Plant Physiol. 147: 636-649.

Ueguchi-Tanaka, M., Ashikari, M., Nakajima, M., Itoh, H., Katoh, E., Kobayashi, M., Chow, T.Y., Hsing, Y.I., Kitano, H., Yamaguchi, I., et al. 2005. GIBBERELLIN INSENSITIVE DWARF1 encodes a soluble receptor for gibberellin. Nature 437: 693-698.

Ueguchi-Tanaka, M., Nakajima, M., Katoh, E., Ohmiya, H., Asano, K., Saji, S., Hongyu, X., Ashikari, M., Kitano, H., Yamaguchi, I., et al. 2007. Molecular interactions of a soluble gibberellin receptor, GID1, with a rice DELLA protein, SLR1, and gibberellin. Plant Cell 19: 2140-2155.

Vieten, A., Sauer, M., Brewer, P.B., and Friml, J. 2007. Molecular and cellular aspects of auxin-transport-mediated development. Trends Plant Sci. 12: 160-168.

Willige, B.C., Ghosh, S., Nill, C., Zourelidou, M., Dohmann, E.M., Maier, A., and Schwechheimer, C. 2007. The DELLA domain of GA INSENSITIVE mediates the interaction with the GA INSENSITIVE DWARF1A gibberellin receptor of Arabidopsis. Plant Cell 19: 1209-1220.

Woodward, A.W. and Bartel, B. 2005. Auxin: Regulation, action, and interaction. Ann. Bot. (Lond.) 95: 707-735.

Xie, D.X., Feys, B.F., James, S., Nieto-Rostro, M., and Turner, J.G. 1998. COI1: An Arabidopsis gene required for jasmonate-regulated defense and fertility. Science 280: 1091-1094.

Xu, L., Liu, F., Lechner, E., Genschik, P., Crosby, W.L., Ma, H., Peng, W., Huang, D., and Xie, D. 2002. The SCF(COI1) ubiquitin-ligase complexes are required for jasmonate response in Arabidopsis. Plant Cell 14: 1919-1935.

Yan, Y., Stolz, S., Chetelat, A., Reymond, P., Pagni, M., Dubugnon, L., and Farmer, E.E. 2007. A downstream mediator in the growth repression limb of the jasmonate pathway. Plant Cell 19: 2470-2483.

Zentella, R., Zhang, Z.L., Park, M., Thomas, S.G., Endo, A. Murase, K., Fleet, C.M., Jikumaru, Y., Nambara, E., Kamiya, Y., et al. 2007. Global analysis of della direct targets in early gibberellin signaling in Arabidopsis. Plant Cell 19: 30373057.

Zhang, D.P., Wu, Z.Y., Li, X.Y., and Zhao, Z.X. 2002. Purification and identification of a 42-kilodalton abscisic acid-specific-binding protein from epidermis of broad bean leaves. Plant Physiol. 128: 714-725. 


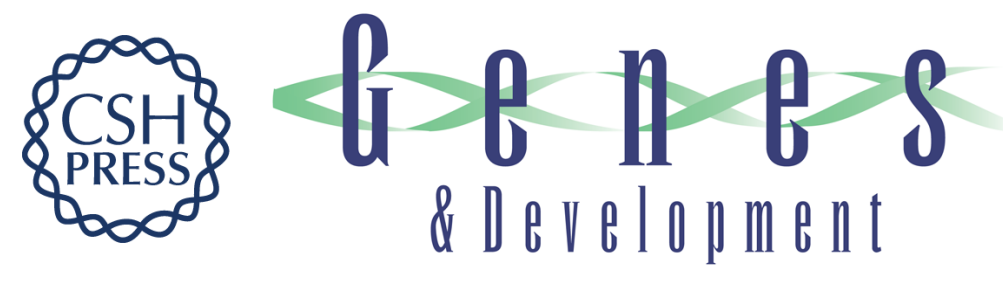

\section{Plant hormone receptors: new perceptions}

Angela K. Spartz and William M. Gray

Genes Dev. 2008, 22:

Access the most recent version at doi:10.1101/gad.1693208

References This article cites 60 articles, 28 of which can be accessed free at: http://genesdev.cshlp.org/content/22/16/2139.full.html\#ref-list-1

License

Email Alerting Receive free email alerts when new articles cite this article - sign up in the box at the top Service right corner of the article or click here.

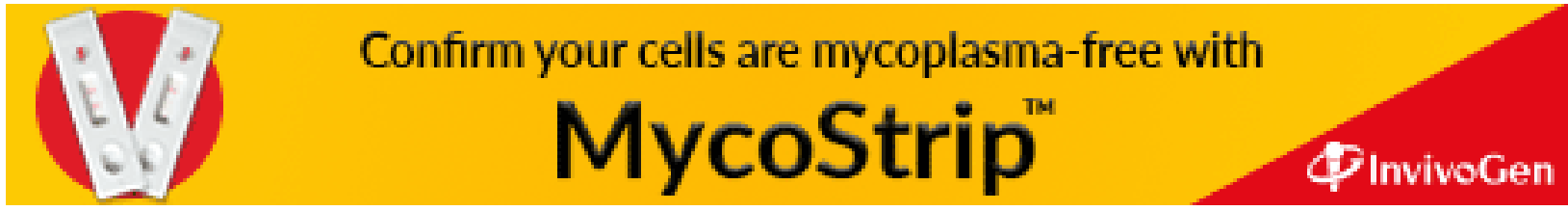

